\title{
Prognostic nutritional index predicts acute kidney injury and mortality of patients in the coronary care unit
}

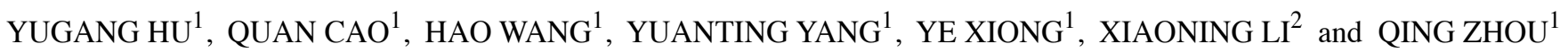 \\ ${ }^{1}$ Department of Ultrasound Imaging, Renmin Hospital of Wuhan University, Wuhan, Hubei 430061; \\ ${ }^{2}$ Department of Nephrology, Zhongnan Hospital of Wuhan University, Wuhan, Hubei 430071, P.R. China
}

Received April 28, 2020; Accepted October 29, 2020

DOI: $10.3892 / e t m .2020 .9555$

\begin{abstract}
The current study aimed to investigate whether prognostic nutritional index (PNI) is an independent predictor of acute kidney injury (AKI) and mortality of patients in the coronary care unit (CCU). In the present two-stage observational study of patients in the CCU, 6,444 patients from the Medical Information Mart for Intensive Care (MIMIC) III database were first enrolled (test cohort), after which 412 patients from Zhongnan Hospital of Wuhan University were recruited in the validation cohort. AKI was defined based on the Kidney Disease Improving Global Outcomes AKI criteria. The primary endpoint was the incidence of AKI stratified by severity, while the second endpoint included in-hospital mortality and 2-year mortality. In the test cohort, 4,457 (69.2\%) patients developed AKI during hospitalization. Following multivariable adjustment, the highest quartile of the PNI value was associated with a 1.8 -fold increased risk of AKI compared with the lowest quartile. For the prediction of AKI, the area under the receiver operating characteristic curve outperformed the acute physiology score III score and clinical model in patients with or without preexisting chronic kidney disease, and this was further validated in the hospital cohort used in the present study. A total of 2,219 patients suffered mortality during the 2-year follow-up, and PNI was indicated to independently predict the risk of in-hospital mortality and 2-year mortality in the test cohort and in the validation cohort. Decision curve analysis indicated that the PNI values were clinically useful; Therefore, the current study
\end{abstract}

Correspondence to: Professor Qing Zhou, Department of Ultrasound Imaging, Renmin Hospital of Wuhan University, 99 Ziyang Road, Wuhan, Hubei 430061, P.R. China

E-mail: qingzhou.wh.edu@hotmail.com

Professor Xiaoning Li, Department of Nephrology, Zhongnan Hospital of Wuhan University, 169 Donghu Road, Wuhan, Hubei 430071, P.R. China

E-mail: xiliusa2000@126.com

Key words: prognostic nutritional index, acute kidney injury, Medical Information Mart for Intensive Care, mortality, coronary care unit demonstrated that the PNI value is an independent predictor of AKI and mortality in patients within the CCU.

\section{Introduction}

Acute kidney injury (AKI), which is one of the most common major complications in patients admitted to hospital and especially those admitted to the intensive care unit worldwide $(1,2)$, is associated with adverse short- and long-term prognosis and increased medical expenses (3). Despite an increasing heterogeneity in its causes and clinical features, detecting patients who are at high risk of AKI may result in earlier diagnosis, avoidance of potentially nephrotoxic exposures and lower health care costs (4,5). A number of biomarkers have been demonstrated to identify patients with an increased risk of AKI and to predict long-term prognosis $(6,7)$. However, the clinical utilization of these biomarkers in patients in the coronary care unit (CCU) is limited.

The prognostic nutritional index (PNI), which is calculated from the serum albumin concentration and total lymphocyte count in the peripheral blood, is an index that reflects chronic inflammation, immune system and nutritional status and indicates prognostic significance in different patients (8). PNI had been described as a simple and objective indicator of adverse outcomes not only in chronic conditions, such as hepatocellular carcinoma (9), chronic heart failure (10) and different cancer types (11), but also in acute illnesses, including acute coronary syndrome (12), acute heart failure (13) and stroke (14). Furthermore, a previous study demonstrated an association between PNI and AKI in patients with normal serum creatinine levels who underwent coronary artery bypass grafting (15). However, to the best of our knowledge, no study has investigated the potential value of PNI for patients in the CCU. The current two-stage observational study aimed to explore and validate the predictive value of PNI for the development of AKI and its prognosis in patients in the CCU.

\section{Materials and methods}

Participants. In the current two-stage observational study, initial data was obtained from the Medical Information Mart for Intensive Care (MIMIC) III database (16). Access to the database was approved by the Institutional Review Board of 
the Massachusetts Institute of Technology and a waiver for informed consent was granted. All adults in the database with a first CCU admission and length of stay $\geq 48 \mathrm{~h}$ were selected $(n=6,903)$. Since MIMIC III is a freely accessible public database, the age of all patients was calculated from the date of admission minus the date of birth. However, for patients $>89$ years old, the date of birth was set to 300 years before their first admission, therefore the actual age of those patients would not be acquired. Moreover, the median age of patients $>89$ years old was 91.4 in this database. Subsequently, these patients were excluded $(n=459)$. Therefore, 6,444 patients constituted the test cohort. A total of 412 adult patients (age, $66.3 \pm 13.1$ years; $32.8 \%$ male) with duration of hospitalization $\geq 48 \mathrm{~h}$ who were admitted to the CCU at Zhongnan Hospital of Wuhan University (Wuhan, China) from January 1, 2014 to June 1,2015 , were prospectively included in the validation cohort, as reported in a previous study (17). The current study was approved by the Ethics Committee of Zhongnan Hospital of Wuhan University and all patients in the validation cohort were required to provide written informed consent.

All laboratory results for the patients enrolled in the test cohort were collected from the MIMICIII database. Since a patient may undergo a single laboratory test more than once during their hospital admission, only the initial test results were included in the final analysis. Patients' baseline characteristics and acute physiology scores III (APSIII) were calculated using the SQL code that was available in the MIMIC code repository, as described in previous studies (18).

PNI value was calculated using the following equation: $10 \mathrm{x}$ serum albumin $(\mathrm{g} / \mathrm{dl})+0.005 \mathrm{x}$ total lymphocyte count $\left(\mathrm{mm}^{3}\right)$. Estimated glomerular filtration rate (eGFR) was calculated using the Chronic Kidney Disease Epidemiology Collaboration (CKD-EPI) equation (19).

The primary outcome was the development of AKI, which was classified according to the Kidney Disease Improving Global Outcomes Clinical Practice Guidelines for AKI based on serum creatinine criteria within 7 days after hospital admission (20), using the SQL code that was available in the MIMIC code repository.

The secondary outcomes included in-hospital mortality and 2-year mortality from admission to the end of the 2-year follow-up period.

Missing data. There were missing data for albumin and lymphocyte counts: $986(15.3 \%)$ patients had a missing albumin variable, and 467 (7.2\%) had a missing lymphocyte count variable in the MIMICIII database. Additionally, 1,269 (19.7\%) patients had a missing PNI value. Imputation for missing variables was considered if they were missing in $>20 \%$ of the patients in the dataset. Predictive mean matching was used to impute numeric features, logistic regression was used for binary variables, and Bayesian polytomous regression was used for factor features.

Statistical analysis. R software (version 3.6.1, http://www. r-project.org) was used for all statistical analyses. Unpaired two-sample t-test or the Mann-Whitney U test was used to compare continuous variables across groups and Pearson's Chi-squared test $\left(\chi^{2}\right)$ to compare categorical variables across groups. Spearman's correlation coefficients were calculated for the correlation between PNI and other biochemical factors. Adjusted odds ratios (ORs) were calculated for AKI using multiple logistic regression. The Cox proportional hazards model was used to estimate the hazard ratio and its associated $95 \%$ confidence interval $(95 \% \mathrm{CI})$. The selection of covariates was based on known or clinically relevant risk factors of AKI $(21,22)$. The clinical model for AKI and prognosis was adjusted for age, sex, BMI, hypertension, diabetes, CKD, eGFR, hemoglobin, leucocytes, mean arterial pressure, triglycerides, total cholesterol, serum potassium and serum sodium. To evaluate the utility of biomarkers in risk classification, the net reclassification index (NRI) and integrated discrimination improvement (IDI) was determined, as described in previous studies $(23,24)$. Furthermore, a decision curve analysis (DCA) was conducted to determine the clinical usefulness of PNI values in the test cohort and validation cohort. $\mathrm{P}<0.05$ was considered to indicate a statistically significant difference.

\section{Results}

Subject characteristics. A total of 6,856 patients $(6,444$ patients in the test cohort and 412 patients in the validation cohort) were analyzed in the current study. There were $1693(26.3 \%)$ patients and $268(65.0 \%)$ cases primarily diagnosed with acute coronary syndrome in the test cohort and in the validation cohort, respectively. Patients were split into groups of those with and those without AKI. In the test cohort, patients who developed AKI were indicated to be older, exhibited more comorbidities (CKD, hypertension and diabetes), higher serum levels of leukocyte, serum albumin, PNI and higher APSIII scores, and lower levels of mean arterial pressure (MAP), serum creatinine, eGFR, hemoglobin and serum albumin compared with those who did not develop AKI. However, the sex distribution, BMI, lymphocyte counts, serum sodium, total cholesterol and triglycerides were comparable between the two groups. Patients enrolled in the validation cohort also exhibited characteristics similar to those in the test cohort except for leukocyte numbers (Table I).

Correlation between PNI and other parameters. The Spearman correlation coefficients indicated that PNI values were significantly positively correlated with leukocyte counts $(r=0.053)$, MAP levels ( $r=0.057)$, eGFR ( $r=0.047)$, hemoglobin levels $(\mathrm{r}=0.051)$ and BMI $(\mathrm{r}=0.032)$. Baseline PNI values were negatively correlated with age $(r=-0.103)$ and APSIII scores ( $\mathrm{r}=-0.077)$. However, no correlations between PNI and triglyceride or total cholesterol were observed (Table II).

PNI as a predictor of the primary endpoint. In the test cohort, AKI occurred in a total of 4,457 patients (69.2\%) within 7 days after admission (Table I). The PNI value was lower in the patients with AKI than in the non-AKI patients (Table I), which was supported by the results of the multivariate analyses. PNI value was associated with incident AKI in patients with or without preexisting chronic kidney disease (CKD) after adjustment for clinical variables in the test cohort. The highest quartile of PNI was associated with an increased risk for AKI by 1.8-fold compared with the lowest quartile in all participants (Table III). When PNI was analyzed as a 
Table I. Characteristics of the CCU patients on admission.

\begin{tabular}{|c|c|c|c|c|c|c|}
\hline \multirow[b]{2}{*}{ Characteristic } & \multicolumn{3}{|c|}{ Test set $(n=6,444)$} & \multicolumn{3}{|c|}{ Validation set $(n=412)$} \\
\hline & No AKI $(n=1,987)$ & AKI $(n=4,457)$ & P-value & No AKI $(n=282)$ & AKI $(n=130)$ & P-value \\
\hline Age, year & $63.6 \pm 13.6$ & $68.6 \pm 15.2$ & $<0.001$ & $65.5 \pm 13.1$ & $68.2 \pm 13.1$ & $<0.001$ \\
\hline Sex, male, n (\%) & $762(38.3)$ & $1,845(38.0)$ & 0.851 & $93(33.0)$ & $42(32.3)$ & 0.893 \\
\hline $\mathrm{BMI}, \mathrm{kg} / \mathrm{m}^{2}$ & $27.9 \pm 4.1$ & $28.0 \pm 6.3$ & 0.652 & $23.8 \pm 3.5$ & $23.7 \pm 3.3 .2$ & 0.870 \\
\hline \multicolumn{7}{|l|}{ Primary diagnosis, n (\%) } \\
\hline Acute coronary syndrome & $503(25.3)$ & $1,190(26.7)$ & 0.243 & $179(63.5)$ & $89(68.5)$ & 0.325 \\
\hline \multicolumn{7}{|l|}{ Preexisting diseases, n (\%) } \\
\hline CKD & $207(10.5)$ & $1,247(28.0)$ & $<0.001$ & $17(6.0)$ & $42(32.3)$ & 0.001 \\
\hline Hypertension & $1,089(54.8)$ & $2,669(59.9)$ & $<0.001$ & $118(41.8)$ & $90(69.2)$ & $<0.001$ \\
\hline Diabetes & 405 (20.4) & $1,140(25.6)$ & $<0.001$ & $79(28.0)$ & $63(48.5)$ & $<0.001$ \\
\hline \multicolumn{7}{|l|}{ Biochemical data } \\
\hline MAP, mmHg & $58.4 \pm 16.7$ & $56.3 \pm 12.7$ & $<0.001$ & $94.6 \pm 13.0$ & $87.8 \pm 13.4$ & $<0.001$ \\
\hline Leukocyte, $\times 10^{9} / 1$ & $12.0 \pm 4.9$ & $13.2 \pm 5.0$ & $<0.001$ & $8.9 \pm 2.8$ & $9.2 \pm 2.3$ & 0.550 \\
\hline Lymphocyte count, $\mathrm{x} 10^{9} / 1$ & $1.6 \pm 0.6$ & $1.5 \pm 0.8$ & 0.565 & $1.5 \pm 0.78$ & $1.4 \pm 0.5$ & 0.178 \\
\hline Hemoglobin, g/l & $127.6 \pm 31.9$ & $109.6 \pm 22.3$ & $<0.001$ & $130.0 \pm 18.6$ & $109.1 \pm 23.4$ & $<0.001$ \\
\hline $\mathrm{eGFR}, \mathrm{ml} / \mathrm{min} / 1.73 \mathrm{~m}^{2}$ & $72.9 \pm 17.7$ & $50.3 \pm 10.4$ & $<0.001$ & $86.3 \pm 10.7$ & $57.1 \pm 15.3$ & $<0.001$ \\
\hline Serum albumin, g/l & $34.2 \pm 2.6$ & $31.9 \pm 2.6$ & $<0.001$ & $38.1 \pm 4.1$ & $35.7 \pm 5.0$ & $<0.001$ \\
\hline Serum creatinine, umol/l & $84.2 \pm 19.2$ & $120.1 \pm 18.1$ & $<0.001$ & $70.9 \pm 15.4$ & $108.4 \pm 12.9$ & $<0.001$ \\
\hline PNI & $56.6 \pm 15.0$ & $46.6 \pm 20.6$ & $<0.001$ & $48.9 \pm 5.3$ & $41.4 \pm 6.0$ & $<0.001$ \\
\hline Total cholesterol, mmol/1 & $3.9 \pm 1.2$ & $4.0 \pm 1.0$ & 0.740 & $4.2 \pm 0.9$ & $4.0 \pm 1.0$ & 0.085 \\
\hline Triglycerides, mmol/1 & $1.2 \pm 0.6$ & $1.1 \pm 0.5$ & 0.364 & $1.7 \pm 1.3$ & $1.5 \pm 1.2$ & 0.243 \\
\hline APACHEII, points & - & - & - & $6.8 \pm 1.8$ & $10.4 \pm 2.2$ & $<0.001$ \\
\hline Serum sodium, mmol/l & $139.5 \pm 3.6$ & $139.5 \pm 4.4$ & 0.537 & $138 \pm 4.8$ & $137 \pm 5.7$ & 0.450 \\
\hline Serum potassium, mmol/1 & $4.5 \pm 0.8$ & $4.7 \pm 0.9$ & $<0.001$ & $3.9 \pm 0.5$ & $4.1 \pm 0.7$ & 0.001 \\
\hline APSIII, points & $32.5 \pm 10.1$ & $47.1 \pm 19.3$ & $<0.001$ & - & - & - \\
\hline LOS, days & $7.1 \pm 2.6$ & $12.0 \pm 4.5$ & $<0.001$ & $11.0 \pm 5.6$ & $16.2 \pm 9.9$ & $<0.001$ \\
\hline Hospital mortality & $445(22.4)$ & $1,747(39.2)$ & $<0.001$ & $17(6.0)$ & $44(33.8)$ & $<0.001$ \\
\hline 2-year mortality & $696(35.0)$ & $2,454(55.1)$ & $<0.001$ & $42(14.9)$ & $70(53.8)$ & $<0.001$ \\
\hline
\end{tabular}

CKD, chronic kidney disease; AKI, acute kidney injury; MAP, mean arterial pressure; eGFR, estimate glomerular filtration rate; PNI, prognostic nutritional index; APACHEII, acute physiology and chronic health evaluation II; APSIII, acute physiology score III; LOS, length of hospital stay; CCU, coronary care unit.

Table II. Correlations between baseline PNI and selected clinical parameters.

\begin{tabular}{lrr}
\hline & \multicolumn{2}{c}{ PNI } \\
\cline { 2 - 3 } Variables & r-value & P-value \\
\hline Age, years & -0.103 & $<0.001$ \\
Hemoglobin, g/l & 0.051 & $<0.001$ \\
BMI, kg/m 2 & 0.032 & 0.021 \\
Leucocyte, $x 109 / 1$ & 0.053 & $<0.001$ \\
Total cholesterol, $\mathrm{mg} / \mathrm{dl}$ & -0.009 & 0.697 \\
Triglycerides, $\mathrm{mg} / \mathrm{dl}$ & -0.012 & 0.705 \\
eGFR, ml/min $/ 1.73 \mathrm{~m}{ }^{2}$ & 0.047 & $<0.001$ \\
MAP, mmHg & 0.057 & $<0.001$ \\
APSIII, points & -0.077 & $<0.001$ \\
\hline
\end{tabular}

PNI, prognostic nutritional index; APSIII, acute physiology score III; eGFR, estimate glomerular filtration rate; MAP, mean arterial pressure. continuous variable, higher PNI values were also associated with the development of AKI (OR=0.982; 95\% CI, 0.975-0.988; $\mathrm{P}<0.001$; data not shown) in a multivariable model. Moreover, lower PNI values group $(<48.8)$ had a higher risk of AKI by 1.3-fold compared with the higher PNI group after adjusting for other risk factors (data not shown).

Performance of PNI for predicting AKI in subgroup analyses. For the prediction of AKI, the area under the receiver operating characteristic curve (AUC) of PNI on admission for all participants in the test set was 0.755 . A cutoff of 48.8 yielded good specificity (81.8\%) and sensitivity (63.5\%; Fig. 1A). The AUCs of PNI in subgroups with and without preexisting CKD were greater compared with APSIII scores, eGFR and the clinical model (Fig. 1B and C; all $\mathrm{P}<0.05$ ). To further validate the predictive value of PNI for AKI, the AUC for predicting AKI was analyzed in an independent validation cohort recruited from Zhongnan Hospital of Wuhan University. As presented in Fig. 1D, PNI was validated in predicting $\mathrm{AKI}$ in the validation cohort $(\mathrm{AUC}=0.738)$. 
Table III. Multivariate logistic regression analyses of PNI as a predictor for AKI in the test cohort.

A, All study participants $(n=6,444)$

\begin{tabular}{lccr}
\hline PNI on admission & Unadjusted OR & Adjusted OR ${ }^{\mathrm{a}}$ & 95\% CI \\
\hline Quartile 1 $(>61.0)$ & 1.0 (ref.) & 1.0 (ref.) & \\
Quartile 2 (42.6-61.0) & 1.652 & 1.414 & $1.170-1.708$ \\
Quartile 3 (34.1-42.5) & 1.799 & 1.447 & $1.195-1.753$ \\
Quartile 4 $(<34.0)$ & 1.897 & 1.765 & $1.457-2.137$ \\
\hline
\end{tabular}

B, Patients without preexisting CKD $(n=4,990)$

\begin{tabular}{lcccr}
\hline PNI on admission & Unadjusted OR & Adjusted OR & 95\% CI & P-value \\
\hline Quartile 1 $(>53.5)$ & $1.0($ ref. & $1.0($ ref. & & \\
Quartile 2 $(43.1-53.5)$ & 2.229 & 1.817 & $1.484-2.226$ & $<0.001$ \\
Quartile 3 $(34.0-43.0)$ & 2.398 & 1.852 & $1.484-2.226$ & $<0.001$ \\
Quartile 4 $(<34.0)$ & 3.084 & 2.262 & $1.820-2.811$ & $<0.001$ \\
\hline
\end{tabular}

C, Patients with preexisting CKD $(n=1,454)$

\begin{tabular}{lccr}
\hline PNI on admission & Unadjusted OR & Adjusted OR ${ }^{\mathrm{a}}$ & 95\% CI \\
\hline Quartile 1 (>55.0) & 1.0 (ref.) & 1.0 (ref.) & 0.001 \\
Quartile 2 (41.6-55.0) & 2.394 & 2.215 & $1.358-3.613$ \\
Quartile 3 (33.0-41.5) & 2.457 & 2.389 & $1.469-3.883$ \\
Quartile 4 $(<33.0)$ & 2.492 & 2.506 & $1.528-4.110$ \\
\hline
\end{tabular}

${ }^{a}$ Adjusted for age, sex, BMI, hypertension, diabetes, eGFR, hemoglobin, leucocyte, mean arterial pressure, triglyceride, total cholesterol, potassium, sodium and APSIII points. OR, odds ratio; 95\% CI, 95\% confidence interval; CKD, chronic kidney disease; eGFR, estimate glomerular filtration rate; PNI, prognostic nutritional index; AKI, acute kidney injury.

PNI as a predictor for secondary endpoints. Of the 6,444 participants in the test cohort, 2,219 (34.4\%) suffered mortality within 2 years after admission. Lower PNI levels were associated with a higher risk of in-hospital mortality [hazard ratio $(\mathrm{HR})=0.978$ (test cohort) and 0.882 (validation cohort)] and 2-year mortality $[\mathrm{HR}=0.984$ (test cohort) and 0.906 (validation cohort); Table IV]. Other parameters, including APACHEII score, eGFR, MAP and age, associated with a higher risk of in-hospital mortality and 2-year mortality are also presented in Table IV. Moreover, a PNI level $<48.8$ on admission was associated with a significantly increased probability of in-hospital mortality $(\mathrm{HR}=1.40 ; 95 \% \mathrm{CI}: 1.27-1.55)$ and 2-year mortality (HR=1.21; 95\% CI, 1.09-1.34; $\mathrm{P}<0.001)$ over the 2-year follow-up period in both cohorts (Fig. 2A and B). In the pre-specified subgroup analysis, patients with PNI $<48.8$ had a higher risk of mortality than those with PNI $\geq 48.8$ in all subgroups except for AKI stages (Fig. 2C and D). Furthermore, a lower level of PNI value (PNI <48.8) on admission was associated with a significantly increased risk of sepsis $(\mathrm{OR}=1.53,95 \% \mathrm{CI}$, 1.32-1.77; $\mathrm{P}<0.001)$ and acute respiratory distress syndrome (ARDS) $(\mathrm{OR}=1.52,95 \% \mathrm{CI}, 1.04-2.22 ; \mathrm{P}=0.029)$ after adjusting for other clinical risk factors (data not shown).

Effect of PNI on risk reclassification of AKI and mortality. To determine whether PNI materially improved risk reclassification, NRI and IDI were used in the test cohort. As presented in Table V, the addition of PNI significantly improved the risk reclassification (as measured using NRI and IDI) of AKI and mortality compared to the APSIII score and clinical model alone.

Clinical usefulness of PNI. To evaluate the clinical use of PNI, a DCA was introduced. According to the DCA, when the threshold probability for a patient was within the range of $0-100 \%$, the PNI added more net benefit than the 'treat all' or 'treat none' strategies both in the test cohort and in the validation cohort (Figs. 3 and S1).

\section{Discussion}

In the test cohort of 6,444 patients, it was revealed that PNI measured on the first day of admission was an independent predictor of AKI in patients in the CCU. The best cutoff value of 48.8 was a good threshold for the risk of mortality in almost all subgroups. The performance of PNI was superior to that of the APSIII scores and the clinical model in the test cohort. Furthermore, the risk reclassification, as measured by the NRI and IDI, was significantly improved through the addition of the APSIII score to the clinical model. The predictive value of PNI was further demonstrated in the validation cohort of 

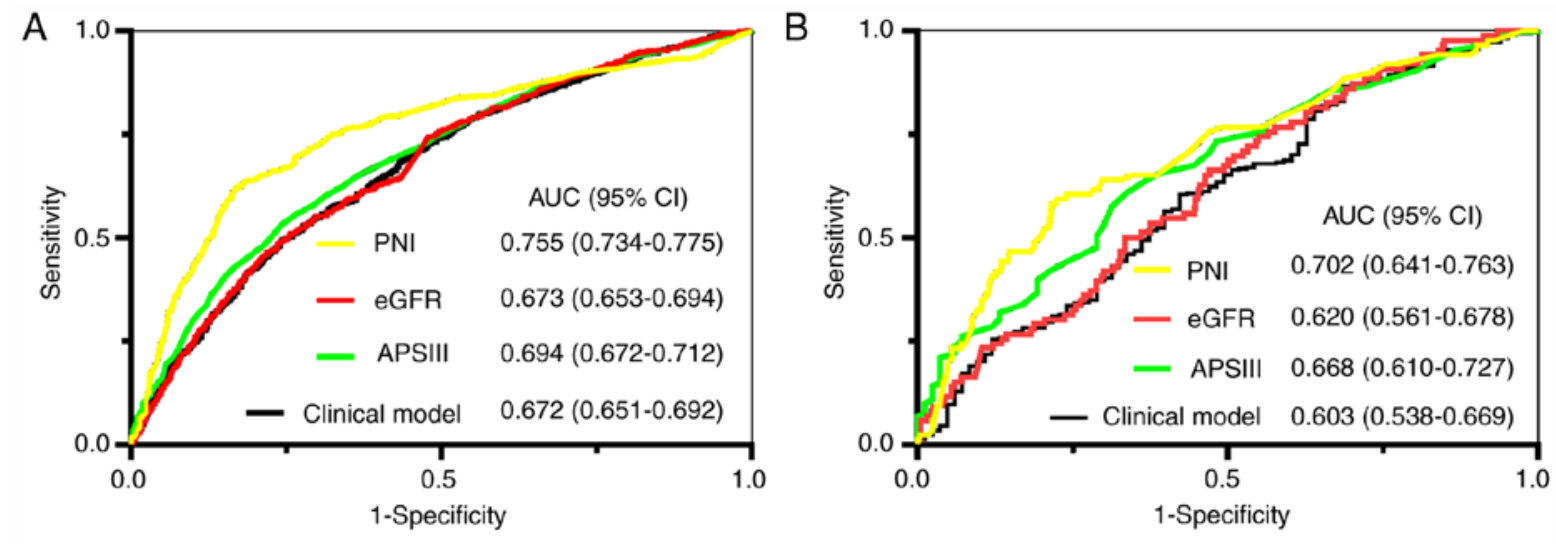

Prediction of $\mathrm{AKI}$ in all participants

\begin{tabular}{cccc}
\hline Marker & Best cutoff & Sensitivity & Specificity \\
\hline PNI & 48.8 & 63.5 & 81.8 \\
APSIII & 43.5 & 55.6 & 73.5 \\
eGFR & 64.1 & 74.3 & 52.2 \\
\hline
\end{tabular}

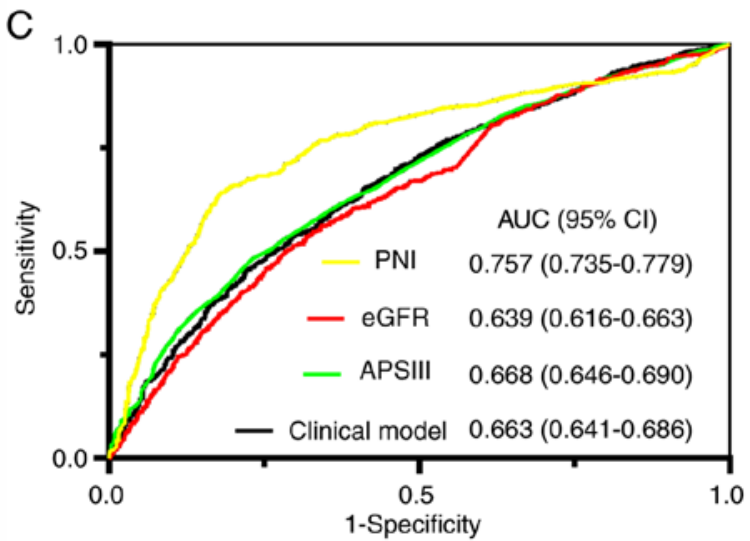

\begin{tabular}{cccc}
\multicolumn{4}{l}{ Prediction of $\mathrm{AKI}$ in participants with CKD } \\
\hline Marker & Best cutoff & Sensitivity & Specificity \\
\hline PNI & 47.3 & 59.3 & 77.4 \\
APSIII & 49.5 & 60.8 & 66.3 \\
eGFR & 30.5 & 74.4 & 45.0 \\
\hline
\end{tabular}

D

Prediction of AKI in participants without CKD

\begin{tabular}{cccc}
\hline Marker & Best cutoff & Sensitivity & Specificity \\
\hline PNI & 58.8 & 65.6 & 80.5 \\
APSIII & 43.5 & 48.2 & 76.9 \\
eGFR & 76.1 & 56.2 & 65.8 \\
\hline
\end{tabular}

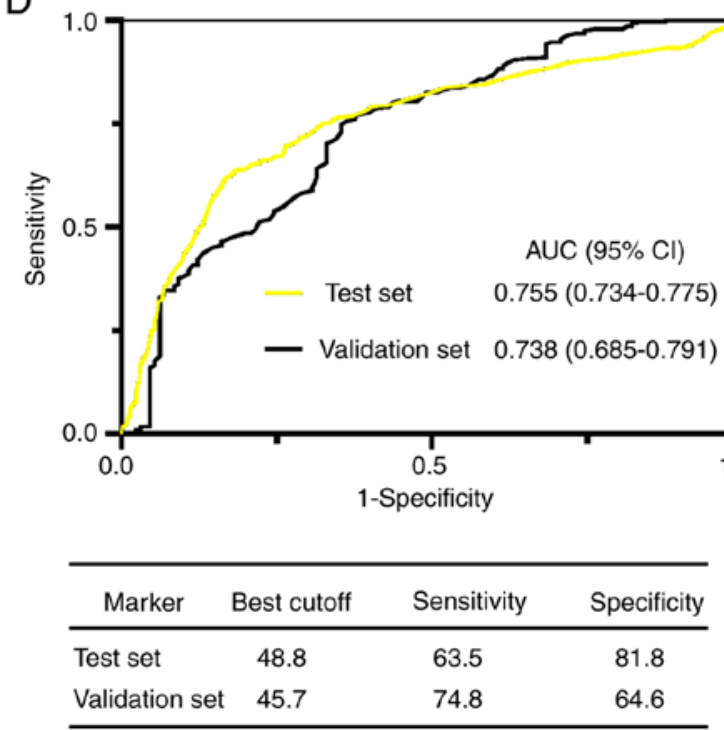

Figure 1. ROC analyses for predicting AKI. (A-C) PNI, eGFR, APSIII and clinical model for predicting AKI in (A) all participants, (B) in patients with preexisting CKD, and (C) in patients without preexisting CKD. (D) ROC analysis for the test and validation sets. ROC, receiver operator characteristic; AKI, acute kidney injury; PNI, prognostic nutritional index; eGFR, Estimated glomerular filtration rate; APSIII, acute physiology scores III; CKD, chronic kidney disease; CI, confidence interval; AUC, area under the curve.

$412 \mathrm{CCU}$ patients. These data suggest that the PNI may be a good predictor for identifying patients at high risk of AKI and mortality in the CCU.

To the best of our knowledge, only two studies have assessed the predictive value of PNI for AKI risk. Dolapoglu et al (15) conducted a retrospective study of 336 consecutive patients with normal serum creatinine levels who underwent coronary artery bypass grafting. The aforementioned study concluded that PNI was independently predictive of AKI in a multivariate logistic regression $(\mathrm{OR}=0.83 ; 95 \% \mathrm{CI}, 0.78-0.88)$. A similar conclusion was drawn in another retrospective study of 423 patients following donor liver transplantation (25). However, to the best of our knowledge, the current study is the first to investigate the association between PNI levels and AKI in a public database, demonstrating that each decrease of a score of 1 in PNI led to a 1.8\% risk of AKI. The results were also independently verified in the hospital cohort.

The association between PNI and CKD has been demonstrated in previous studies. Hori et al (26) revealed an association between decreased PNI value $(<54)$ and lower postoperative renal function after 12 months of donor nephrectomy in a retrospective observational study of 75 living kidney donors. The aforementioned study further concluded that the decreased PNI value independently predicted the development of CKD G3b (OR=0.3; 95\% CI, 0.1-0.8). Similarly, a positive correlation between PNI value and eGFR ( $r=0.047)$ was indicated in the current study based on Spearman's correlation coefficient. Furthermore, a previous study that investigated the impact of PNI on cardiovascular disease (CVD) mortality 
Table IV. Multivariate Cox regression analyses: Predictors of hospital mortality and 2-year mortality in test and validation sets.

A, in-hospital mortality

\begin{tabular}{|c|c|c|c|c|}
\hline \multirow[b]{2}{*}{ Parameter } & \multicolumn{2}{|c|}{ Test set } & \multicolumn{2}{|c|}{ Validation set } \\
\hline & Adjusted HR (95\% CI) & P-value & Adjusted HR (95\% CI) & P-value \\
\hline APACHEII score & - & - & $1.121(1.040-1.208)$ & 0.003 \\
\hline PNI & $0.978(0.975-0.981)$ & $<0.001$ & $0.882(0.845-0.921)$ & $<0.001$ \\
\hline $\mathrm{MAP}, \mathrm{mmHg}$ & 0.977 (0.964-0.989) & $<0.001$ & $0.986(0.974-0.999)$ & 0.034 \\
\hline APSIII score & $1.003(1.001-1.006)$ & 0.016 & - & - \\
\hline $\mathrm{eGFR}, \mathrm{ml} / \mathrm{min}$ & $0.994(0.992-0.996)$ & $<0.001$ & $0.982(0.955-1.010)$ & 0.210 \\
\hline
\end{tabular}

B, 2-year mortality

\begin{tabular}{|c|c|c|c|c|}
\hline \multirow[b]{2}{*}{ Parameter } & \multicolumn{2}{|c|}{ Test set } & \multicolumn{2}{|c|}{ Validation set } \\
\hline & Adjusted HR (95\% CI) & P-value & Adjusted HR (95\% CI) & P-value \\
\hline APACHEII score & - & - & $1.145(1.080-1.214)$ & $<0.001$ \\
\hline PNI & $0.984(0.983-0.986)$ & $<0.001$ & $0.906(0.877-0.936)$ & $<0.001$ \\
\hline MAP, mmHg & $0.974(0.965-0.982)$ & $<0.001$ & $0.983(0.973-0.993)$ & 0.001 \\
\hline APSIII score & $1.021(1.017-1.024)$ & $<0.001$ & - & - \\
\hline $\mathrm{eGFR}, \mathrm{ml} / \mathrm{min}$ & $0.997(0.995-0.999)$ & 0.006 & $1.001(0.988-1.014)$ & 0.855 \\
\hline Age, years & $1.014(1.012-1.016)$ & $<0.001$ & $1.002(0.980-1.025)$ & 0.857 \\
\hline Preexisting CKD & $1.223(1.098-1.363)$ & $<0.001$ & $1.008(0.548-1.856)$ & 0.979 \\
\hline Hypertension & $1.255(1.155-1.365)$ & $<0.001$ & $1.166(0.762-1.783)$ & 0.480 \\
\hline
\end{tabular}

Multivariate model for in-hospital mortality and 2-year mortality are adjusted for age, sex, BMI, hypertension, diabetes, CKD, eGFR, hemoglobin, leucocyte, mean arterial pressure, triglyceride, total cholesterol, serum potassium and serum sodium and APACHEII or APSIII points. HR, hazard ratio; CI, confidence interval; APACHEII, acute physiology and chronic health evaluation II; PNI, prognostic nutritional index; MAP, mean arterial pressure; APSIII, acute physiology score III; eGFR, estimate glomerular filtration rate; CKD, chronic kidney disease.

in patients with incident peritoneal dialysis concluded that PNI may be a better predictor of CVD mortality than hemoglobin and leukocytes (27). A similar association was also indicated in patients undergoing continuous ambulatory peritoneal dialysis (CAPD). Cai et al (28) conducted a retrospective cohort study of 1,501 patients with CAPD and found a robust and consistent association between lower PNI value $(<45)$ and overall mortality or CVD mortality that was independent of other common risk factors $(\mathrm{HR}=1.82 ; 95 \% \mathrm{CI}$, 1.36-2.43; HR=1.63; 95\% CI, 1.06-2.51). Additionally, PNI was a prognostic factor in patients undergoing kidney transplantation and in patients undergoing peritoneal dialysis $(25,29)$. The results of the current study similarly suggested that PNI admission was a significant determinant of mortality, indicating that each decrease by a score of 1 in PNI led to a $2.2 \%$ increase in the risk of in-hospital mortality and $1.6 \%$ increase in the risk of 2-year mortality.

Up to $80 \%$ of hospitalized patients are malnourished worldwide (30), especially critically ill patients, as they may lose $10-25 \%$ of their body protein content within 10 days after admission (31). Serum albumin, which is a common indicator of nutritional status, was an independent predictor for the development of postoperative AKI and mortality in a retrospective study of 2,339 patients who underwent aneurysm clipping surgery (32). Additionally, a previous study demonstrated that nutritional intervention may improve clinical outcomes with shorter hospital stays and lower costs (33). In the current study, PNI was correlated with the known indicators of nutritional status, including BMI and hemoglobin. Furthermore, patients in the CCU are often in a heightened proinflammatory state, which can significantly worsen nutritional status (34), and AKI is known to be associated with intrarenal and systemic inflammation (35). Consequently, PNI, combined serum albumin and total lymphocyte count, which represent nutritional status and chronic inflammation $(36,37)$, may be indicated for risk stratification and clinical management for patients in the CCU. In the present study, it was demonstrated that PNI, which is clinically and easily available, was an independent predictor for the development of AKI and prognosis in patients in the CCU. In the subgroup analyses, PNI $<48.8$ exhibited a higher risk of 2-year mortality in almost all subgroups. These findings were then further validated in the hospital cohort. Moreover, in the current study, the AUCs of PNI for AKI were 0.755 in the test cohort and 0.738 in the validation cohort, which were consistent with previous models for AKI in different populations $(38,39)$.

The present study had a few limitations. There was a sizeable number of missing data related to PNI and given the 
A

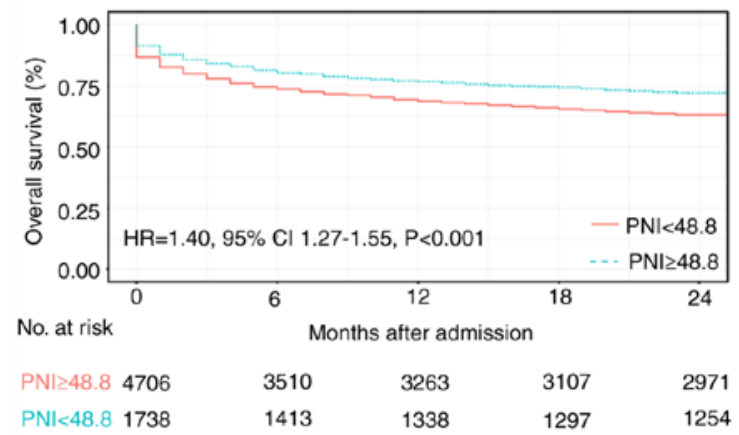

C

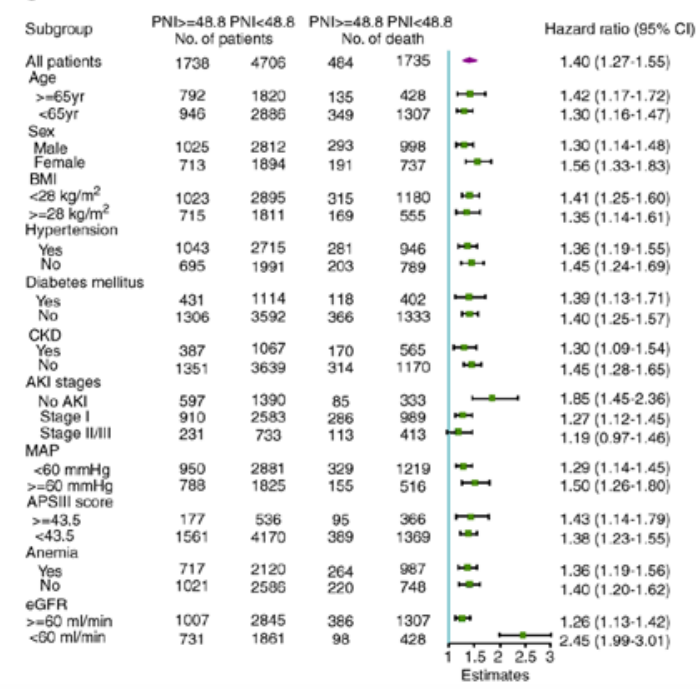

B

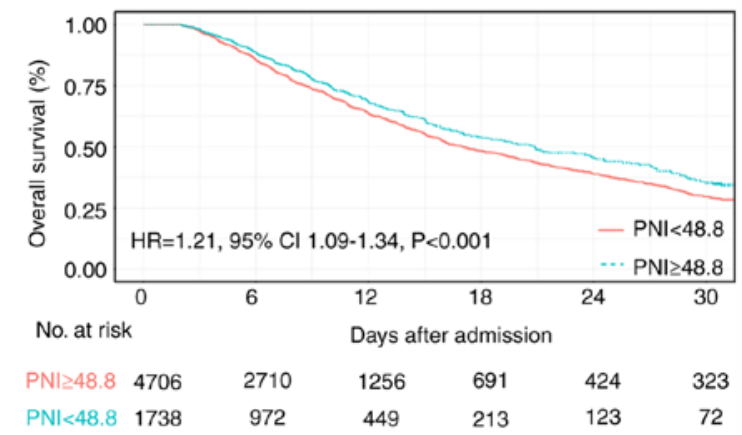

D

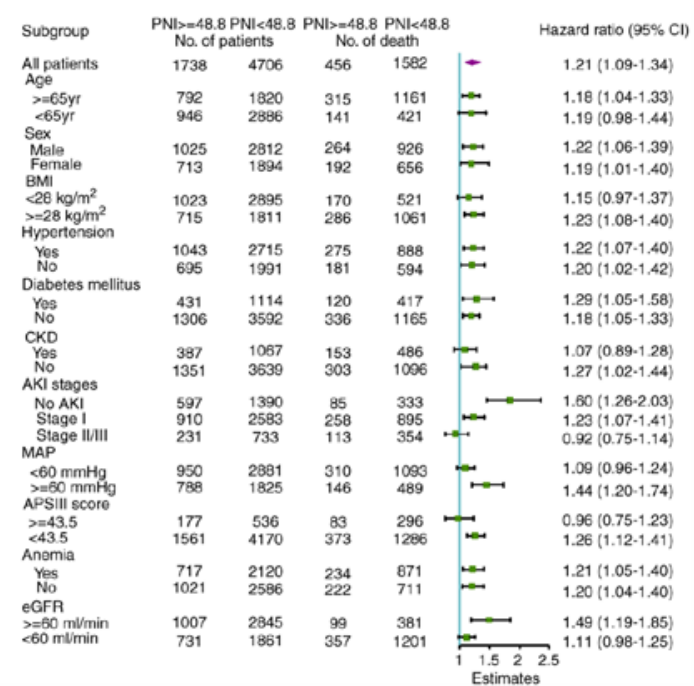

Figure 2. PNI levels and mortality risk in coronary care unit patients. Kaplan-Meier estimates of (A) 2-year survival and (B) in-hospital survival. Subgroup analyses at the second interim analysis for (C) 2-year mortality and (D) in-hospital mortality in the test cohort. Hazard ratios and number of deaths among high-risk and low-risk patients are presented. HR, hazard ratio; CI, confidence interval; PNI, prognostic nutritional index; CKD, chronic kidney disease; AKI, acute kidney injury; APSIII, acute physiology scores III; MAP, mean arterial pressure; eGFR, Estimated glomerular filtration rate.

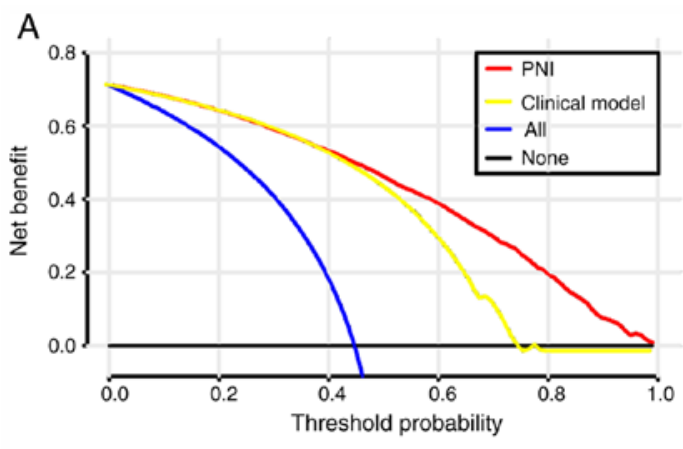

B
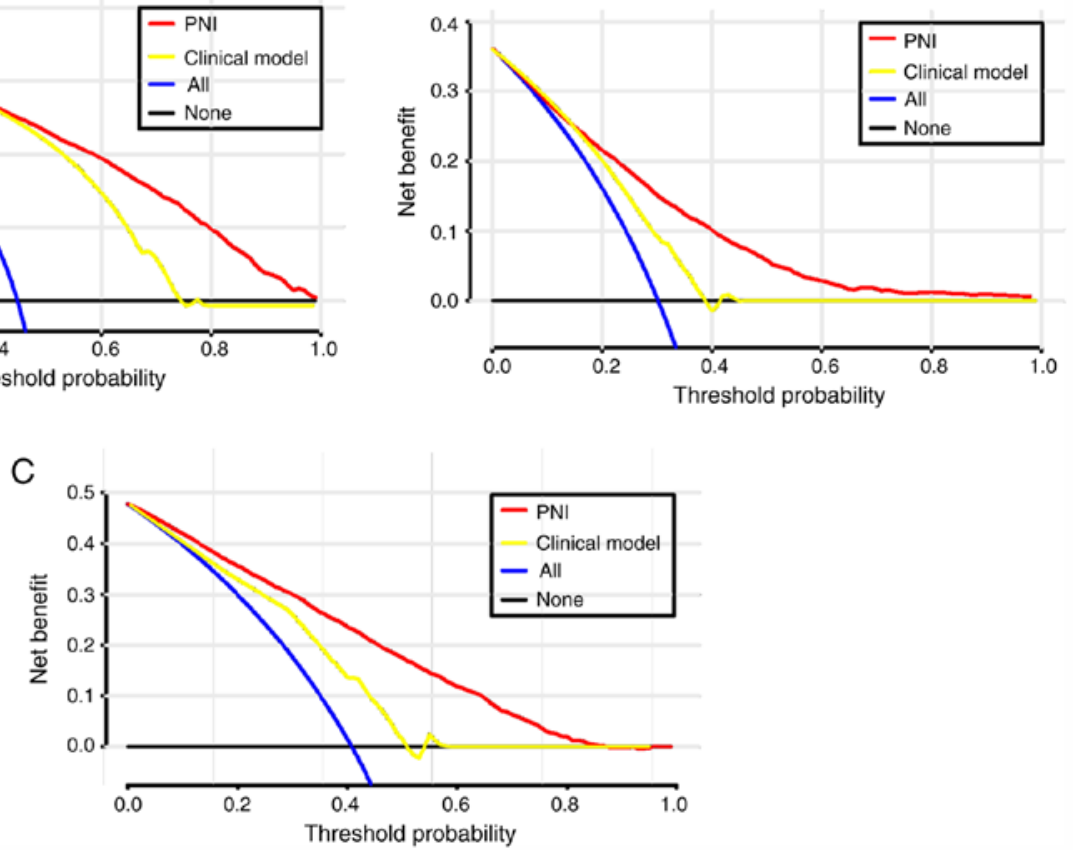

Figure 3. DCA for PNI value and clinical model to detect its clinical usefulness in the test cohort. (A) The DCA of PNI and clinical model for the development of AKI; (B) the DCA of PNI and clinical model for in-hospital mortality; (C) the DCA of PNI and clinical model for 2-year mortality. DCA, Decision curves analysis; PNI, prognostic nutritional index. 
Table V. NRI and IDI analyses for risk reclassification of AKI and mortality in test cohort.

A, AKI

\begin{tabular}{|c|c|c|c|c|c|c|c|c|}
\hline \multirow[b]{2}{*}{ Outcome } & \multicolumn{4}{|c|}{ AUC } & \multicolumn{2}{|l|}{ IDI } & \multicolumn{2}{|c|}{$\mathrm{NRI}^{\mathrm{a}}$} \\
\hline & Biomarker & $\begin{array}{l}\text { Biomarker+ } \\
\text { clinical model }\end{array}$ & $\begin{array}{l}\text { Clinical } \\
\text { model }^{\mathrm{b}}\end{array}$ & P-value & $\begin{array}{c}\text { Value } \\
(95 \% \mathrm{CI})\end{array}$ & P-value & $\begin{array}{c}\text { Value } \\
(95 \% \mathrm{CI})\end{array}$ & P-value \\
\hline PNI & 0.755 & 0.787 & 0.672 & 0.003 & $\begin{array}{c}0.044 \\
(0.020-0.117)\end{array}$ & $<0.001$ & $\begin{array}{c}0.169 \\
(0.086-0.382)\end{array}$ & $<0.001$ \\
\hline APSIII & 0.694 & 0.730 & & 0.279 & $\begin{array}{c}0.021 \\
(0.017-0.076)\end{array}$ & $<0.001$ & $\begin{array}{c}0.014 \\
(-0.071-0.101)\end{array}$ & 0.737 \\
\hline PNI+APSIII & 0.784 & 0.801 & & $<0.001$ & $\begin{array}{c}0.712 \\
(0.047-0.158)\end{array}$ & $<0.001$ & $\begin{array}{c}0.199 \\
(0.120-0.408)\end{array}$ & $<0.001$ \\
\hline
\end{tabular}

$\mathrm{B}$, In hospital mortality

\begin{tabular}{|c|c|c|c|c|c|c|c|c|}
\hline \multirow[b]{2}{*}{ Outcome } & \multicolumn{4}{|c|}{ AUC } & \multicolumn{2}{|l|}{ IDI } & \multicolumn{2}{|c|}{$\mathrm{NRI}^{\mathrm{a}}$} \\
\hline & Biomarker & $\begin{array}{l}\text { Biomarker+ } \\
\text { clinical model }\end{array}$ & $\begin{array}{l}\text { Clinical } \\
\text { model }^{\mathrm{b}}\end{array}$ & P-value ${ }^{c}$ & $\begin{array}{c}\text { Value } \\
(95 \% \mathrm{CI})\end{array}$ & P-value & $\begin{array}{c}\text { Value } \\
(95 \% \mathrm{CI})\end{array}$ & $\mathrm{P}$-value \\
\hline PNI & 0.737 & 0.788 & 0.663 & $<0.001$ & $\begin{array}{c}0.015 \\
(-0.011-0.253)\end{array}$ & 0.066 & $\begin{array}{c}0.177 \\
(-0.030-0.238)\end{array}$ & 0.134 \\
\hline APSIII & 0.686 & 0.716 & & 0.001 & $\begin{array}{c}0.037 \\
(-0.030-0.140)\end{array}$ & 0.294 & $\begin{array}{c}0.068 \\
(-0.150-0.332)\end{array}$ & 0.420 \\
\hline PNI+APSIII & 0.779 & 0.804 & & $<0.001$ & $\begin{array}{c}0.124 \\
(0.022-0.244)\end{array}$ & 0.028 & $\begin{array}{c}0.192 \\
(0.007-0.404)\end{array}$ & 0.046 \\
\hline
\end{tabular}

C, 2-year mortality

\begin{tabular}{|c|c|c|c|c|c|c|c|c|}
\hline \multirow[b]{2}{*}{ Outcome } & \multicolumn{4}{|c|}{ AUC } & \multicolumn{2}{|c|}{ IDI } & \multicolumn{2}{|c|}{$\mathrm{NRI}^{\mathrm{a}}$} \\
\hline & Biomarker & $\begin{array}{l}\text { Biomarker+ } \\
\text { clinical model }\end{array}$ & $\begin{array}{l}\text { Clinical } \\
\text { model }^{\mathrm{b}}\end{array}$ & P-value & $\begin{array}{c}\text { Value } \\
(95 \% \mathrm{CI})\end{array}$ & P-value & $\begin{array}{c}\text { Value } \\
(95 \% \mathrm{CI})\end{array}$ & $\mathrm{P}$-value \\
\hline PNI & 0.735 & 0.780 & 0.683 & $<0.001$ & $\begin{array}{c}0.047 \\
(0.021-0.137)\end{array}$ & 0.002 & $\begin{array}{c}0.123 \\
(-0.009-0.356)\end{array}$ & 0.058 \\
\hline APSIII & 0.684 & 0.711 & & 0.003 & $\begin{array}{c}0.024 \\
(0.003-0.103)\end{array}$ & 0.024 & $\begin{array}{c}0.095 \\
(-0.068-0.286)\end{array}$ & 0.236 \\
\hline PNI+APSIII & 0.777 & 0.797 & & $<0.001$ & $\begin{array}{c}0.072 \\
(0.033-0.158)\end{array}$ & 0.002 & $\begin{array}{c}0.213 \\
(0.037-0.387)\end{array}$ & 0.026 \\
\hline
\end{tabular}

${ }^{a}$ The NRI is calculated through two-way category by using the event rate of AKI and mortality in the test cohort. ${ }^{b}$ The clinical model for predicting AKI and mortality are composed of age, sex, BMI, hypertension, diabetes, CKD, eGFR, total cholesterol, hemoglobin, leucocyte, mean arterial pressure, triglyceride, serum potassium and serum sodium. ${ }^{\circ}$ Biomarker+clinical model versus clinical model. AUC, area under the receiver-operating characteristic curve; IDI, integrated discrimination improvement; NRI, Net reclassification index; PNI, prognostic nutritional index; APSIII, acute physiology score III; CI, confidence interval; AKI, acute kidney injury.

possible selection bias, the affected patients were not excluded, which may have led to oversights in the analysis. Additionally, PNI values were calculated on the first day of admission but changes in PNI were not assessed in the patients during their hospital stay and their 2-year follow-up. The initial results also may not represent patient situation, and the maximum or minimum results may have been more suitably compared with the initial results. However, for prediction models, the initial results may be more appropriate to predict outcomes as clinicians are able to rapidly classify the risk of AKI and prognosis. Moreover, some of the data requires further analysis as MAP was much higher in the validation cohort compared with the test cohort, which may reflect organ perfusion. In previous studies, a target MBP up to $80-95 \mathrm{mmHg}$ has been 
associated with favorable outcomes, including a reduction in renal failure and mortality for critically ill patients (40). Lower MAP has also been indicated to be an independent predictor for AKI in a previous study (41). In the present study, patient blood pressure was collected upon CCU admission and a lower MAP may indicate worse organ perfusion, which can lead to a higher incidence of AKI. Finally, some factors such as troponin, brain natriuretic peptide and the usage of drugs and markers of inflammation were not assessed in the clinical model. Therefore, further studies are required to validate the results of the current study.

In conclusion, the current study demonstrated that PNI values could serve as an early predictor for the development of AKI and mortality in patient in CCU, and PNI on admission exhibited good predictive performance and may be a useful clinical marker that can be used for estimating long-term survival in these patients. These results were validated in a hospital cohort. If the results are further confirmed in future studies, given that PNI is a readily available and cost-effective parameter, its use as an index to stratify the risk of AKI and mortality is suggested to be promising.

\section{Acknowledgements}

Not applicable.

\section{Funding}

No funding was received.

\section{Availability of data and materials}

The datasets used and/or analyzed during the current study are available from the corresponding author on reasonable request.

\section{Authors' contributions}

YH contributed to the conception and design of this work, acquisition of data, analysis and interpretation of the data, and drafting of the discussion, QC, HW, YY and YX contributed to interpretation of the data and drafting of the discussion. QZ and XL are the guarantors of this work, contributed to the conception and design of this work, and have full access to all of the data in the current study. All authors read and approved the final manuscript.

\section{Ethics approval and consent to participate}

Access to the MIMIC-III database for research was approved by the Institutional Review Boards of the Massachusetts Institute of Technology (Cambridge, MA, USA) and the Beth Israel Deaconess Medical Center, and was granted a waiver of informed consent. Due to the HIPAA-compliant deidentification in the MIMIC-III database, the institutional IRB requirement was waived. The current study was performed in accordance with the ethical standards laid down in the 1964 Declaration of Helsinki and its later amendments. Furthermore, the current study was also approved by the Ethics Committee of Zhongnan Hospital of Wuhan University. All patients in the validation cohort were required to provide written informed consent.

\section{Patient consent for publication}

Not applicable.

\section{Competing interests}

The authors declare that they have no competing interests.

\section{References}

1. Bellomo R, Kellum JA, Ronco C, Wald R, Martensson J, Maiden M, Bagshaw SM, Glassford NJ, Lankadeva Y, Vaara ST and Schneider A: Acute kidney injury in sepsis. Intensive Care Med 43: 816-828, 2017.

2. Santos RPD, Carvalho ARS, Peres LAB, Ronco C and Macedo E: An epidemiologic overview of acute kidney injury in intensive care units. Rev Assoc Med Bras (1992) 65: 1094-1101, 2019.

3. Abd ElHafeez S, Tripepi G, Quinn R, Naga Y, Abdelmonem S, AbdelHady M, Liu P, James M, Zoccali C and Ravani P: Risk, predictors, and outcomes of acute kidney injury in patients admitted to intensive care units in Egypt. Sci Rep 7: 17163, 2017.

4. Hobson C, Ozrazgat-Baslanti T, Kuxhausen A, Thottakkara P, Efron PA, Moore FA, Moldawer LL, Segal MS and Bihorac A: Cost and mortality associated with postoperative acute kidney injury. Ann Surg 261: 1207-1214, 2015.

5. Collister D, Pannu N, Ye F, James M, Hemmelgarn B, Chui B, Manns B and Klarenbach S; Alberta Kidney Disease Network: Health care costs associated with AKI. Clin J Am Soc Nephrol 12: 1733-1743, 2017.

6. Greenberg JH, Zappitelli M, Jia Y, Thiessen-Philbrook HR, de Fontnouvelle CA, Wilson FP, Coca S, Devarajan P and Parikh CR: Biomarkers of AKI progression after pediatric cardiac surgery. J Am Soc Nephrol 29: 1549-1556, 2018.

7. da Rocha EP, Yokota LG, Sampaio BM, Cardoso Eid KZ, Dias DB, de Freitas FM, Balbi AL and Ponce D: Urinary neutrophil gelatinase-associated lipocalin is excellent predictor of acute kidney injury in septic elderly patients. Aging Dis 9: 182-191, 2018.

8. Hong X, Cui B, Wang M, Yang Z, Wang L and Xu Q: Systemic immune-inflammation index, based on platelet counts and neutrophil-lymphocyte ratio, is useful for predicting prognosis in small cell lung cancer. Tohoku J Exp Med 236: 297-304, 2015.

9. Wang D, Hu X, Xiao L, Long G, Yao L, Wang Z and Zhou L: Prognostic nutritional index and systemic immune-inflammation index predict the prognosis of patients with HCC. J Gastrointest Surg: Feb 5, 2020 (Epub ahead of print).

10. Zencirkiran Agus H and Kahraman S: Prognostic nutritional index predicts one-year outcome in heart failure with preserved ejection fraction. Acta Cardiol 75: 450-455, 2020.

11. Salati M, Filippi R, Vivaldi C, Caputo F, Leone F, Salani F, Cerma K, Aglietta M, Fornaro L, Sperti E, et al: The prognostic nutritional index predicts survival and response to first-line chemotherapy in advanced biliary cancer. Liver Int 40: 704-711, 2020.

12. Cheng Y, Li H, Li D, Liang L, Jia Y, Zou L, Li F, Zhu X, Qian H, He N, et al: Prognostic nutritional index may not be a good prognostic indicator for acute myocardial infarction. Sci Rep 9: 14717, 2019.

13. Cheng YL, Sung SH, Cheng HM, Hsu PF, Guo CY, Yu WC and Chen $\mathrm{CH}$ : Prognostic nutritional index and the risk of mortality in patients with acute heart failure. J Am Heart Assoc 6: e004876, 2017.

14. Scrutinio D, Lanzillo B, Guida P, Passantino A, Spaccavento S and Battista P: Association between malnutrition and outcomes in patients with severe ischemic stroke undergoing rehabilitation. Arch Phys Med Rehabil 101: 852-860, 2020.

15. Dolapoglu A, Avci E, Kiris T and Bugra O: The predictive value of the prognostic nutritional index for postoperative acute kidney injury in patients undergoing on-pump coronary bypass surgery. J Cardiothorac Surg 14: 74, 2019.

16. Johnson AE, Pollard TJ, Shen L, Lehman LW, Feng M, Ghassemi M, Moody B, Szolovits P, Celi LA and Mark RG: MIMIC-III, a freely accessible critical care database. Sci Data 3: 160035,2016

17. Hu Y, Liu H, Fu S, Wan J and Li X: Red blood cell distribution width is an independent predictor of AKI and mortality in patients in the coronary care unit. Kidney Blood Press Res 42: 1193-1204, 2017. 
18. Singh P, Pathak S and Sharma RM: A comparison of acute physiology and chronic health evaluation III and simplified acute physiology score II in predicting sepsis outcome in intensive care unit. Anesth Essays Res 12: 592-597, 2018.

19. Schwandt A, Denkinger M, Fasching P, Pfeifer M, Wagner C, Weiland J, Zeyfang A and Holl RW: Comparison of MDRD, CKD-EPI, and cockcroft-gault equation in relation to measured glomerular filtration rate among a large cohort with diabetes. J Diabetes Complications 31: 1376-1383, 2017.

20. Kellum JA and Lameire N: Diagnosis, evaluation, and management of acute kidney injury: A KDIGO summary (Part 1). Crit Care 17: 204, 2013.

21. Singbartl K and Kellum JA: AKI in the ICU: Definition, epidemiology, risk stratification, and outcomes. Kidney Int 81: 819-825, 2012 .

22. Zhang Y, Jiang L, Wang B and Xi X: Epidemiological characteristics of and risk factors for patients with postoperative acute kidney injury: A multicenter prospective study in 30 Chinese intensive care units. Int Urol Nephrol 50: 1319-1328, 2018.

23. Pencina MJ, D'Agostino RB Sr, D'Agostino RB Jr and Vasan RS Evaluating the added predictive ability of a new marker: From area under the ROC curve to reclassification and beyond. Stat Med 27: 157-172, 207-212, 2008.

24. Cook NR: Statistical evaluation of prognostic versus diagnostic models: Beyond the ROC curve. Clin Chem 54: 17-23, 2008

25. Min JY, Woo A, Chae MS, Hong SH, Park CS, Choi JH and Chung HS: Predictive impact of modified-prognostic nutritional index for acute kidney injury within 1-week after living donor liver transplantation. Int J Med Sci 17: 82-88, 2020.

26. Hori S, Miyake M, Morizawa Y, Nakai Y, Onishi K, Iida K, Gotoh D, Anai S, Torimoto K, Aoki K, et al: Impact of preoperative abdominal visceral adipose tissue area and nutritional status on renal function after donor nephrectomy in Japanese living donors for renal transplantation. Ann Transplant 23: 364-376, 2018.

27. Peng F, Chen W, Zhou W, Li P, Niu H, Chen Y, Zhu Y and Long H: Low prognostic nutritional index associated with cardiovascular disease mortality in incident peritoneal dialysis patients. Int Urol Nephrol 49: 1095-1101, 2017.

28. Cai L, Yu J, Yu J, Peng Y, Ullah H, Yi C, Lin J, Yang X and $\mathrm{Yu} \mathrm{X}$ : Prognostic value of inflammation-based prognostic scores on outcome in patients undergoing continuous ambulatory peritoneal dialysis. BMC Nephrol 19: 297, 2018.

29. Chen KH, Wu CH, Hsu CW, Chen YM, Weng SM, Yang CW and Hung CC: Protein nutrition index as a function of patient survival rate in peritoneal dialysis. Kidney Blood Press Res 33 174-180, 2010

30. Hartz LLK, Stroup BM, Bibelnieks TA, Shockey C and Ney DM: ThedaCare nutrition risk screen improves the identification of non-intensive care unit patients at risk for malnutrition compared with the nutrition risk screen 2002. JPEN J Parenter Enteral Nutr 43: 70-80, 2019.

31. Puthucheary ZA, Rawal J, McPhail M, Connolly B, Ratnayake G, Chan P, Hopkinson NS, Phadke R, Dew T, Sidhu PS, et al: Acute skeletal muscle wasting in critical illness. JAMA 310: 1591-1600, 2013.
32. Bang JY, Kim SO, Kim SG, Song JG, Kang J, Kim JW and Ha S: Impact of the serum albumin level on acute kidney injury after cerebral artery aneurysm clipping. PLoS One 13: e206731, 2018.

33. Piggott KD, Liu A, Monczka J, Fakioglu H, Narasimhulu SS, Pourmoghadam $\mathrm{K}$ and DeCampli W: Inadequate preoperative nutrition might be associated with acute kidney injury and greater illness severity postoperatively. J Thorac Cardiovasc Surg 155: 2104-2109, 2018.

34. White JV, Guenter P, Jensen G, Malone A and Schofield M; Academy of Nutrition and Dietetics Malnutrition Work Group; A.S.P.E.N. Malnutrition Task Force; A.S.P.E.N. Board of Directors: Consensus statement of the academy of nutrition and Dietetics/American society for parenteral and enteral nutrition: Characteristics recommended for the identification and documentation of adult malnutrition (undernutrition). J Acad Nutr Diet 112: 730-738, 2012

35. Rabb H, Griffin MD, McKay DB, Swaminathan S, Pickkers P, Rosner MH, Kellum JA and Ronco C; Acute Dialysis Quality Initiative Consensus XIII Work Group: Inflammation in AKI: Current understanding, key questions, and knowledge gaps. J Am Soc Nephrol 27: 371-379, 2016.

36. Zhang H, Tao Y, Wang Z and Lu J: Evaluation of nutritional status and prognostic impact assessed by the prognostic nutritional index in children with chronic kidney disease. Medicine (Baltimore) 98: e16713, 2019.

37. Itami Y, Miyake M, Tatsumi Y, Gotoh D, Hori S, Morizawa Y, Iida K, Ohnishi K, Nakai Y, Inoue T, et al: Preoperative predictive factors focused on inflammation-, nutrition-, and muscle-status in patients with upper urinary tract urothelial carcinoma undergoing nephroureterectomy. Int J Clin Oncol 24: 533-545, 2019.

38. Kalisvaart M, Schlegel A, Umbro I, de Haan JE, Polak WG, IJzermans JN, Mirza DF, Perera MTP, Isaac JR, Ferguson J, et al: The AKI prediction Score: A new prediction model for acute kidney injury after liver transplantation. HPB (Oxford) 21: 1707-1717, 2019.

39. Patidar KR, Xu C, Shamseddeen H, Cheng YW, Ghabril MS, Mukthinuthalapati VVPK, Fricker ZP, Akinyeye S, Nephew LD, Desai AP, et al: Development and validation of a model to predict acute kidney injury in hospitalized patients with cirrhosis. Clin Transl Gastroenterol 10: e00075, 2019.

40. Asfar P, Meziani F, Hamel JF, Grelon F, Megarbane B, Anguel N, Mira JP, Dequin PF, Gergaud S, Weiss N, et al: High versus low blood-pressure target in patients with septic shock. N Engl J Med 370: 1583-1593, 2014.

41. Kwon HM, Moon YJ, Jung KW, Jeong HW, Park YS, Jun IG, Song JG and Hwang GS: Low mean arterial blood pressure is independently associated with postoperative acute kidney injury after living donor liver transplantation: A propensity score weighing analysis. Ann Transplant 23: 236-245, 2018.

This work is licensed under a Creative Commons Attribution-NonCommercial-NoDerivatives 4.0 International (CC BY-NC-ND 4.0) License. 